\title{
Preemption Window mechanism for efficient QoS support in E-OBS network architecture
}

\author{
Davide Careglio*, Miroslaw Klinkowski* ${ }^{\dagger}$, Josep Solé-Pareta* \\ *Advanced Broadband Communication Center \\ Universitat Politècnica de Catalunya, Barcelona, Catalunya, 08034 Spain \\ Email: \{careglio, mklinkow, pareta\}@ac.upc.edu \\ ${ }^{\dagger}$ National Institute of Telecommunications \\ Warsaw, 04-894 Poland
}

\begin{abstract}
This paper focuses on the problem of quality of service (QoS) provisioning in optical burst switching (OBS) networks. OBS is a promising photonic network technology aiming at efficient transport of IP traffic by means of statistical multiplexing. The lack of optical memories, however, makes this operation quite complicated. Problems such as unfairness in access to the shared transmission resources, facility in adopting alternative and backup routing, scheduling complexity and so on arise in the conventional OBS architecture. In [1] we proposed the offset-time emulated OBS (E-OBS) architecture, which overcomes all these drawbacks by means of distributed provisioning of the offset time in core nodes. Nonetheless it is still difficult to guarantee a certain level of service quality. Burst preemption mechanism, which, alongside with offset-time differentiation, was proven to be the most effective technique for QoS provisioning in OBS networks. The general drawback of any burst preemptionbased mechanism is that, in case of successful preemption, either the resources reserved for the preempted bursts on outgoing path are wasted or an additional signaling procedure should be carried out in order to release them. In order to avoid wasted resources reservation, in [2] we proposed the Preemption Window (PW) mechanism which enhances the E-OBS for efficient QoS support. In this paper we evaluate exhaustively the performance of the resulting architecture showing all its advantageous with respect to other solutions.
\end{abstract}

\section{INTRODUCTION}

Optical burst switching (OBS) is a promising solution for reducing the gap between switching and transmission speeds in future networks [3]. Packets coming from client networks are aggregated and assembled into optical data bursts in the edge nodes of an OBS network. A burst control packet (BCP) is transmitted through a dedicated control channel and delivered prior to the data burst (the so called offset-time). In this way the electronic controller of an intermediate (core) node has enough time both to reserve a wavelength on its output link, usually for the duration time of the incoming burst, and to reconfigure dynamically the switching matrix. The output wavelength is released for other connections when the burst transmission is finished in the node. Such a temporary utilization of wavelengths allows for higher resource utilization as well as for better adaptation to highly variable input traffic in comparison to optical circuit-switching networks. Moreover the aggregation of data packets helps to overcome the fast processing and switching requirements of optical packet switching (OPS) technology. In fact, OBS allows using state-of-the-art switching elements [4].

There are two distinct signalling architectures considered for OBS networks. The first one is based on a connection-oriented signalling protocol which performs end-to-end resources reservation with acknowledgment in so called two-way reservation mode. The other exploits a connection-less signalling protocol which allocates the resources on-the-fly, a while before the burst arrival, in a one-way reservation mode1. Since the problem of the two-way reservation signalling concerns the latency due to the connection establishment process such architectures are less interesting for long-haul network applications due to the large latency and are not addressed in this paper.

The one-way reservation signalling that can operate effectively in large distance OBS networks performs according to a statistical multiplexing paradigm; hence it encounters the problem of burst contention inside the network. Indeed, when a burst control packet enters a node in order to perform the wavelength reservation for its data burst, it may happen that the requested resources are not available at the output link and the burst has to be dropped. The lack of optical random access memories complicates the resolution of burst contention in optical networks. To alleviate this problem several mechanisms based on wavelength conversion, deflection routing and fibre delay line (FDL) buffering together with dedicated burst scheduling algorithms have been proposed.

From the very beginning, there were two distinct control architectures considered for OBS networks [3]. The difference between them comes from different management of offset times. A conventional OBS (C-OBS) introduces the offset time in soft-way by delaying the transmission of burst with respect to the $\mathrm{BCP}$ in the edge node. At each core node, the offset time decreases by the time the BCP spends in the switch controller. Another idea for an OBS operation comes from OPS world and it intends to emulate the offset time by means of an additional fiber delay unit (FDU) introduced in the data path at the input port of the core node in the so called offset time emulated OBS (E-OBS) architecture. FDU delays the arrival of the burst with respect to the arrival of its $\mathrm{BCP}$ and in such hard-way it introduces the offset-time. Although C-OBS has attracted lots of attention we highlighted in [1] that problems such as unfairness in access to the shared transmission resources, 
facility in adopting alternative and backup routing, scheduling complexity, etc. can be avoided in E-OBS.

In this paper we deal with the problem of Quality of Service (QoS) provisioning in the E-OBS architecture. Effective QoS provisioning engages both the definition of specific QoS classes to be given for higher level applications and dedicated mechanisms providing such classes in the network. QoS mechanisms in OBS networks based on one-way signalling usually utilize a services differentiation approach, which may be exploited in different ways:

- differentiation of the burst inherent parameters in edge nodes such as offset times -in so called Offset-Time Differentiation mechanism [5]- and burst size [6],

- differentiation of reservation and scheduling procedures in core nodes such as threshold-based, burst preemption and intentional burst dropping schemes [7], or

- differentiation of signaling procedures and routing strategies [8].

Burst preemption (BP), offset time differentiation (OTD) and wavelength threshold mechanisms are the most addressed QoS mechanism in OBS networks. In [9] we showed that BP outperforms the other mechanisms in terms of overall throughput while maintaining the same burst loss probability as for OTD for high priority traffic.

The general drawback of preemptive mechanisms is a need for an additional signaling protocol to be used to release resources in case of the successful preemption. Indeed in a COBS architecture (see Section III) even when the preemption happens, the control packet corresponding to preempted burst (the so called phantom burst) continues its trip towards the destination node reserving the resources. In such a case either the resources reserved for the preempted bursts on outgoing path are wasted or an additional signaling procedure should be carried out.

In [2] we proposed the Preemption Window (PW) technique which enhances the E-OBS architecture for QoS support. We proved that the problem of phantom bursts is overtaken using such technique while maintaining the same good performance as for the classical burst preemption.

In this paper we present a deeper analysis of the benefits of the PW technique applied in the E-OBS architecture compared to the burst preemption in C-OBS. The rest of the paper is organized as follows. In Section II we briefly describe the E-OBS network architecture and its benefits with respect to C-OBS one. Section III is dedicated to the presentation of the Preemption Window technique and its behavior. In Section IV, an exhaustive analysis of the performance of the PW technique applied in E-OBS is presented for both single node scenario and network scenario. Section V draws some conclusions.

\section{E-OBS NETWORK ARCHITECTURE}

Figure 1 presents the E-OBS network architecture. An EOBS node is a typical OBS node [10] with additional optical taps to extract the control channels and a pool of fiber delay units (FDUs) introduced into the data path of the input interface -each input fiber is connected to one FDU. Note that

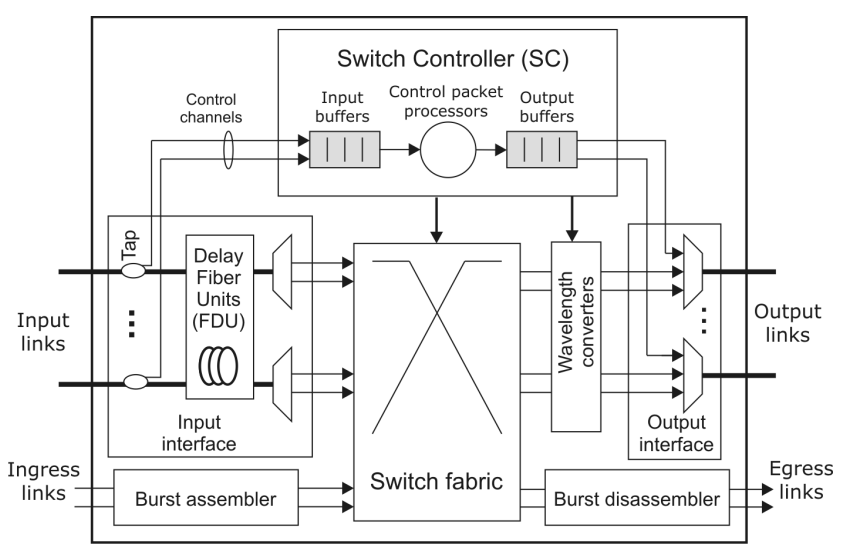

(a)

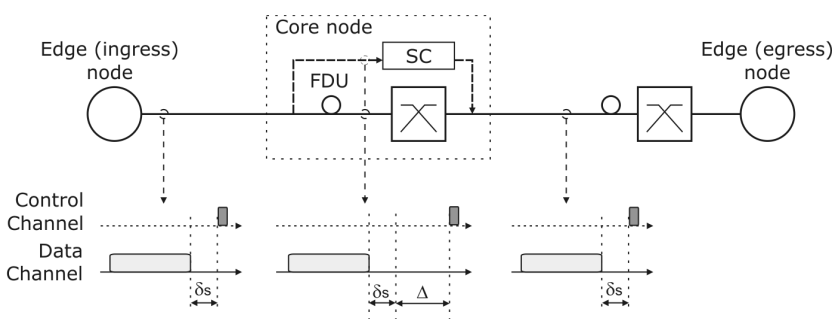

(b)

Fig. 1. a) General E-OBS node architecture and b) example of behavior. $\Delta$ is the 1-hop offset time corresponding to the queuing and processing delay of one node, $\delta_{s}$ is the switching delay

in the literature the FDU term is usually replaced by the FDL term; nevertheless, we use FDU so that to distinguish this component from more complex FDL buffers.

E-OBS architecture allows a different control operations than C-OBS. The edge node launches the BCP into the control channel prior to its data burst and with some small offset time provided to compensate the switch reconfiguration delay at the egress node $\left(\delta_{s}\right.$ in Fig. 1(b)). At each core node, while the BCP goes directly to the switch controller, the data burst is delayed by the FDU for a period $\Delta$ (which depends on the length of the FDU). During this time, the BCP undergoes the queueing in an input buffer and the processing in one (or more) processor unit(s). Before being converted back to optical form and transmitted through the output control channel to the output interface, the BCP is buffered in such a way that the offset time is as it was at the ingress. This operation is repeated at each core node so that the offset is kept as fixed as possible from link to link inside the network. Once the burst reaches the egress node, it is disassembled and the data are delivered to the client networks.

In [1] and [14] we showed that C-OBS posses several drawbacks such as the problem of unfairness in access to transmission resources, constraints in the alternative routing, a need for complex void filling-based resource reservation algorithms, some difficulties in QoS provisioning, etc. On the contrary, thanks to the introduction of one FDU of few km per input port in the core nodes and to its fixed offset provisioning, the E-OBS can bring significant facilities to the mentioned 
problems. At the same time, E-OBS performs as well as COBS in terms of burst loss probability and end-to-end delay.

\section{PREEMPTION Window}

\section{A. The problem of phantom burst in burst preemption}

Burst Preemption (BP) can be classified as a contention resolution based mechanism that in case of contention allows the processing unit of the switch to overwrite a low priority (LP) reservation with a later arriving high priority (HP) one. The preemption may concern either the whole burst [15] (full preemption) or it allows for a partial preemption when a burst segmentation technique [16] is applied. Although burst segmentation offers better performance characteristics it is at the cost of higher complexity since this technique involves additional information about the data bursts to be carried and processed in the core nodes.

As mentioned in Section I, the general drawback of burst preemptive mechanisms is the possible waste of resources on the ongoing path due to the phantom bursts. In COBS networks, the burst control packet which belongs to a preempted LP data burst does not have any knowledge about the preemption. Thus, it continues its trip towards the destination node and consumes unnecessarily both the controlplane resources, when being processed in the node controllers, and data-plane resources, when reserving the wavelengths for its (preempted) data burst.

In order to assess such an overhead, we develop an approximate estimation of the preemption effect that is produced in a single node. In particular, we introduce a preemption rate $(R)$ metric that represents the number of preempted bursts over all the bursts (successfully) transmitted at the node output link.

If we assume i.e.d. burst inter-arrival times and i.i.d. burst lengths, the preemption rate of a full burst preemption scheme can be calculated as (see Appendix A for a derivation):

$$
R=\frac{\alpha_{H P}\left[\operatorname{Erl}(\rho, W)-\operatorname{Erl}\left(\alpha_{H P} \rho, W\right)\right]}{1-\operatorname{Erl}(\rho, W)}
$$

where $\rho, \alpha_{H P}, W$ are, respectively, the overall load, HP class relative load, the number of wavelengths in the link, and $\operatorname{Erl}($.$) is the Erlang B-loss formula given by (9).$

Figure 2 presents the preemption rate of a $\mathrm{BP}$ mechanism in a single node scenario. As we can see, $R$ significantly increases in the systems with lower number of wavelengths as well as at higher traffic loads. A small disparity between analytical and simulation results comes from the fact that the simulated bursts are stream-like arranged in a data channel (bursts can not overlap each other) and their arrivals are not more exponentially distributed.

$R$ corresponds to the percentage of additional signalling required at each node to release the preempted bursts. If such signalling procedure is not provided there is a waste of transmission resources due to these preempted reservations in all the nodes on the ongoing paths. In large networks of high number of nodes the problem might be intensified since all nodes undergo similar effect.

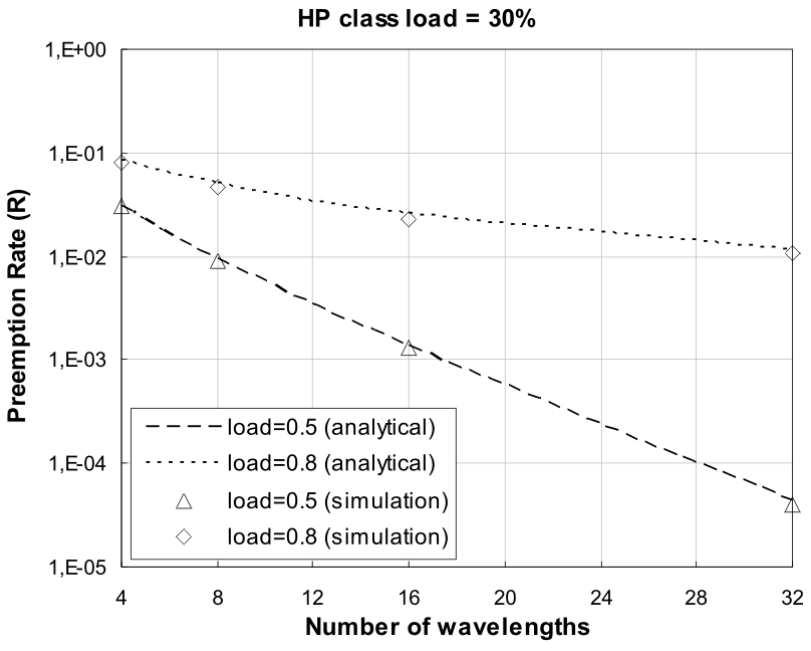

(a)

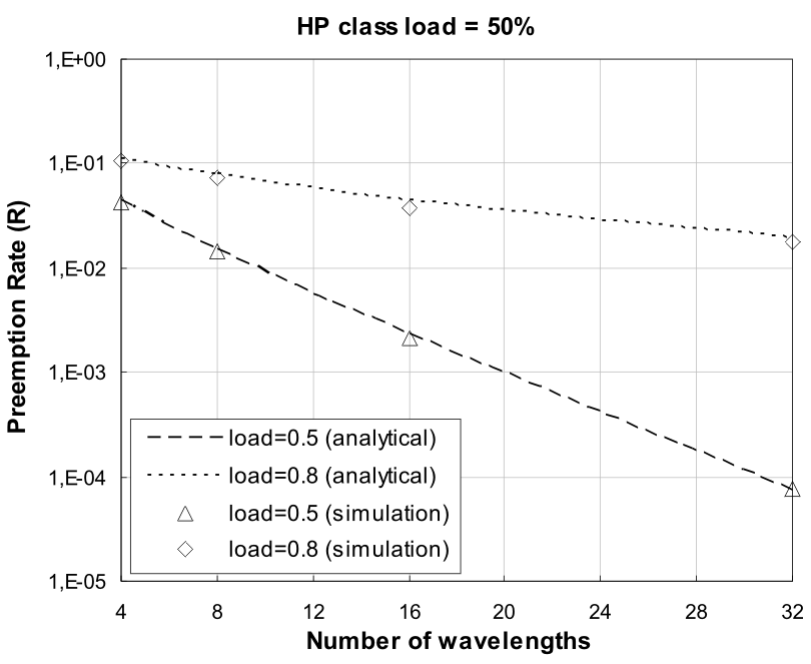

(b)

Fig. 2. Percentage of additional signalling necessary to release preempted burst at each node, with HP class load: a) $30 \%$, b) $50 \%$.

\section{B. The principle of Preemption Window}

Taking into account the reasons explained in the previous section there is a motivation for adapting the E-OBS architecture for the burst preemptive mechanism. In such an architecture there is no offset-time setup by edge nodes. The offset is artificially introduced by means of additional FDU inserted in the data path at the input port of core nodes. Control packet and burst travel simultaneously through the network. When both reach a core node the control packet goes directly to the switch control unit, whilst the burst is delayed in the FDU by period $\Delta$ (the 1-hop offset time). During this time the control packet is processed.

Starting with this basis, E-OBS can be enhanced with the QoS support by means of the Preemption Window (PW) mechanism. In such a mechanism, a control packet is delivered to the switch controller with some extra offset $\left(\Delta_{e}\right)$, besides the 1-hop offset time $(\Delta)$. This additional offset constitutes a 


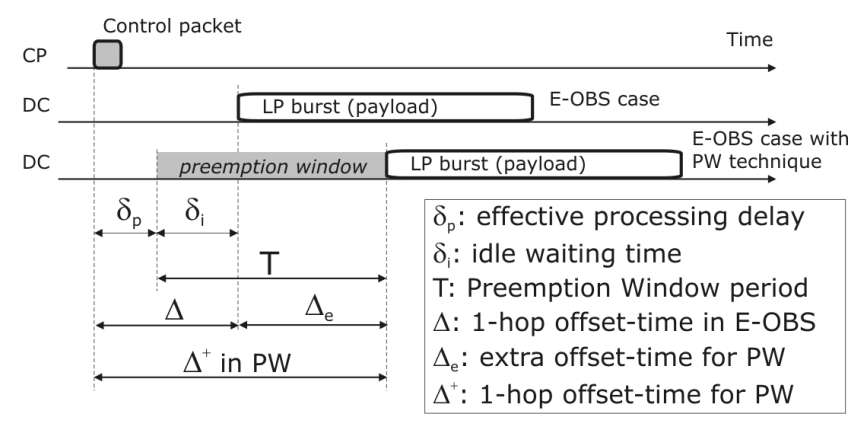

Fig. 3. The length of preemptive window in PW mechanism.

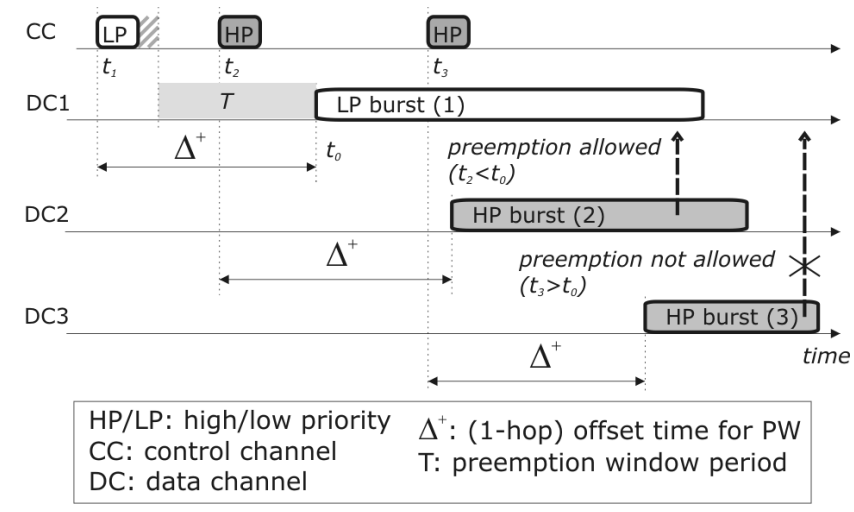

Fig. 4. Principles of the preemption window mechanism.

preemptive window $T$ during which the controller can preempt the reservation of lower priority by the one of higher priority. Preemptive window $T$ begins after the end of processing of the burst control packet and lasts till the arrival of its payload (see Figure 3). In further discussion, for simplicity, we assume that the payload comprises a guard band for the switching operation.

Figure 4 shows an illustrative example of the PW mechanism. In this example, a preemption of the LP burst 1 can be performed only by the HP burst 2 since the control packet of the later arrives in preemptive window $T$. On the other hand, the HP burst 3 is not allowed to preempt the LP burst 1 because its control packet arrives out of window $T$.

An important rule of the $\mathrm{PW}$ mechanism is that the $\mathrm{BCP}$, after its processing, is waiting in the memory of the controller until $T$ expires and only then it can be sent to the next node (if the burst has not been preempted) or dropped (in case of successful preemption). After the BCP is sent the preemption of its burst is not allowed in the node. Thanks to these rules any $\mathrm{BCP}$ has its corresponding data burst (no phantom bursts are present) and there is no need for any signaling procedure to be carried out in order to release the resources on the outgoing path in case of successful burst preemption. It should be pointed out that the PW mechanism can work with both full and partial burst preemption techniques.

The preemption offset can be provided in both C-OBS and E-OBS architectures. In the former the edge node adds an additional offset, which accounts the preemption windows in all the nodes of the routing path. A disadvantage of this solution is the increase of variation of offset times, which may further intensify the unfairness in access to transmission resources. For this reason we consider the PW mechanism is more appropriate for E-OBS architectures.

In the PW mechanism, the value of $T$ becomes an important trade-off between high burst delay (too large preemptive window) and ineffective burst preemption (too short preemptive window). Period $T$ can be calculated as:

$$
T=\Delta^{+}-\delta_{p}
$$

where $\Delta^{+}$is the offset introduced by inlet FDU in E-OBS node, and $\delta_{p}$ is the effective processing delay of control packet.

Since $\delta_{p}$ could be variable, period $T$ could vary as well. In the simplest case, $T$ corresponds to the idle waiting time period $\delta_{i}$ after the processing of control packet. In order to increase this period, the FDU can add some additional preemptive offset $\Delta_{p}$. In this case $T$ could be also expressed as:

$$
T=\delta_{i}+\Delta_{p}
$$

Scope of the following section is to give an overview of the effect of the value of $T$ to the burst loss probability.

\section{NUMERICAL RESULTS}

In this section, we use event-driven simulation to show that a full-burst preemptive mechanism in E-OBS architecture with the PW mechanism applied can achieve easily the performance of classical burst preemption in the conventional OBS. We analyze two different scenarios: in the first one we consider a single node, which can be buffer-less or enhanced with some FDLs capabilities, whilst in the second one a full network scenario with bufferless nodes is considered.

In all cases, two classes of services, namely High Priority (HP) and Low Priority (LP) are available. The metrics that we study are the burst loss probabilities, both for the HP $\left(B L P_{H P}\right)$ and the LP $\left(B L P_{L P}\right)$ class as well as overall BLP $\left(B L P_{\text {Total }}\right)$.

\section{A. Node scenario}

We consider a general non-blocking OBS node architecture with full wavelength conversion. The switch has $4 \times 4$ input/output ports and $W$ number wavelengths per port, each one operating at 10 Gbps. A one-way signaling protocol, the simple Horizon resources reservation, and the LAUC scheduling are applied. The switching and processing times are $1 \mu s$ and $10 \mu s$, respectively.

The traffic is uniformly distributed between all input and output ports. Regarding the burst length and the inter-arrival time (IAT) distributions we apply the ones studied in [17][18]. In particular, the bursts length is Gaussian distributed with a mean burst length equal to 40kbytes $(1 \mu)$. Minimum burst length is setup to 4kbytes while its maximum value is 4Mbytes. The burst IAT, after the assembly process, is also Gaussian distributed with a mean depending on the traffic 
load. The mean load per input channel (wavelength) is 0.8 . The percentage of HP traffic load is denoted as $\alpha$ and it is equal to $25 \%$ if not specified differently.

It is worth to mention that all simulation results have $99 \%$ level of confidence. It is achieved by means of at least 10 repetition of the same simulation.

1) Bufferless node: For the bufferless case, in Fig. 5 we can see that the preemption window, which is equal to $T$, has a big impact on $B L P_{H P}$ characteristics. In particular, when $T$ increases performance of the preemption mechanism improves resulting in lower $B L P_{H P}$. It is due to the fact that the more time the LP burst reservation is exposed to be preempted the higher probability that HP burst preempts it. Other remark is that all the $B L P_{H P}$ characteristics look alike regardless of the system parameters. Indeed the characteristics fall quasilinearly from their maximum obtained for $T=0$ and they slow down rapidly at $T=1.3 * 1 / \mu$, to stabilize at about $2 / \mu$. These results could serve us in order to find an upper bound on the effective offset introduced by means of the input FDU.

The behavior described above can be explained by Fig. 5(a) that presents comparative results of $B L P_{H P}$ for 3 resources reservation mechanisms, namely without preemption (NP), a classical Burst Preemption (BP) when even the preemption of LP burst being transmitted is allowed and finally the PW mechanism. We can see that for $T=0$ which results in the lack of preemption window, the PW offers the same results like the NP. In such case no preemption can occur and indeed the PW and NP behaves the same. On the other side, for $T$ above $2 / \mu$ the PW achieves the performance of the BP mechanism. To go further we have to recall both the considered burst length distribution that is a Gaussian like with a mean equal to $1 / \mu$ and the principle of operation of the PW mechanism. Namely, assuming that preemption window is higher than $2 / \mu$, from the burst length distribution we obtain that almost all bursts are shorter than this preemption window. For this reason all those bursts' reservations are exposed for preemption during whole their duration and this is just like in the BP. Therefore the BP mechanism can be easily emulated by the PW when $T$ is high enough.

From Fig. 5(a) we can also see that the impact of PW mechanism on $B L P_{L P}$ and $B L P_{\text {Total }}$ is small. The $B L P_{L P}$ curve slightly deteriorates when increasing $T$ and it stabilizes soon. With higher $T$ a given LP reservation is exposed for more time to the preemption and the preemption may occur even at the moment being close to the end of reservation. In such case we may waste more resources belonging to this reservation prior to the moment of preemption what can impact the $B L P_{\text {Total }}$. Nevertheless, as the simulation results show this effect is almost imperceptible since $B L P_{\text {Total }}$ is very stable in the whole range of $T$ and it is only slightly deteriorated at higher offset-times in comparison to the NP case (when $T=0$ ).

Figure 5(b) presents the performance obtained for different system and traffic parameters. Briefly, we can notice that increasing the number of wavelength $(\lambda \mathrm{s})$ as well as decreasing the percentage of HP traffic load $(\alpha)$ improves $B L P_{H P}$

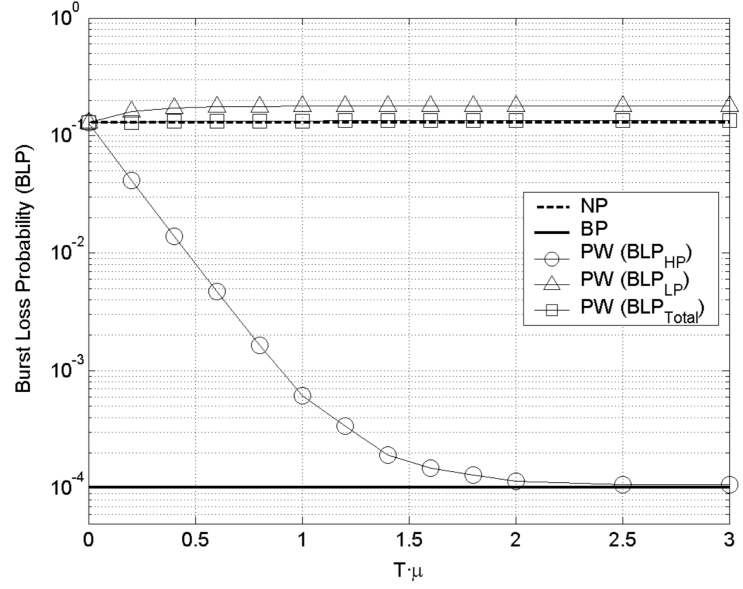

(a)

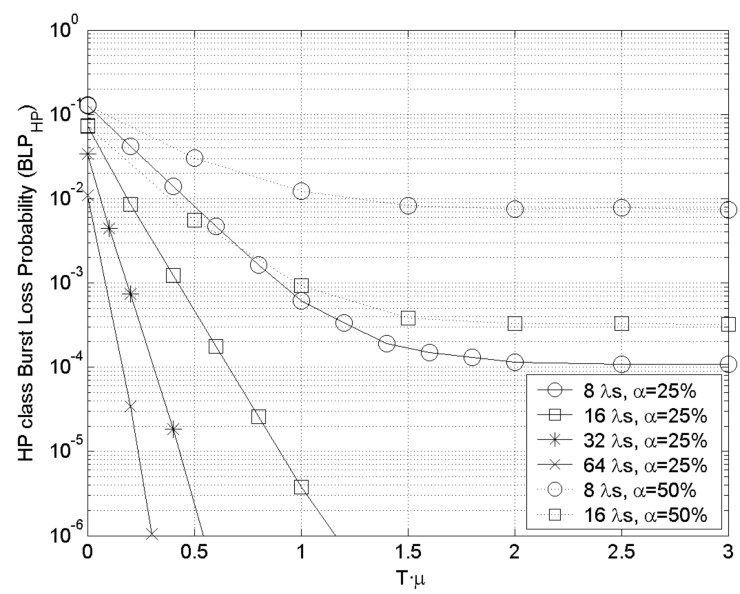

(b)

Fig. 5. a) Burst Loss Probabilities for different reservation mechanisms, b) HP burst loss probability vs. HP traffic ratio and number of wavelengths.

characteristics what seems to be obvious. Moreover, we see again that all the curves become stable starting from $T=2 / \mu$. Figure 5 shows also that effective PW guaranteeing low HP blocking probability (e.g. on the level of $10^{-6}$ ) would be reduced in the systems with more wavelengths.

For the bufferless node, we provided analytical model and results for the case of single wavelength in [1]. In case of multiple wavelengths, additional simulation results are available in [14] where also the exponential traffic model is considered.

2) Node with FDL buffering: For the scenario with buffering capabilities we assume the core node enhanced with a feed-back FDL buffer [19]. Such architecture allows us to preempting any LP burst by a HP burst even if it is actually transmitted through the buffer's FDL. In fact, when preemption occurs we know that thanks to our control architecture (see Section III) the LP burst has not reached the output port. Therefore, we can easily block it by means of the switching matrix in order to make impossible its propagation towards the output link. Note that preemption of a burst being transmitted 


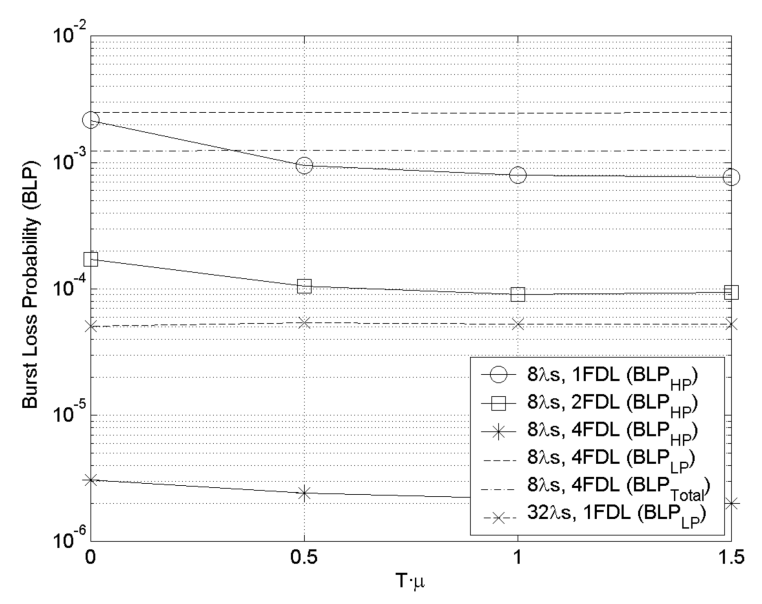

Fig. 6. a) Blocking probabilities in the node with FDL buffers applied $(\alpha=25 \%)$.

through the feed-forward FDL buffer might result in the propagation of a part of optical signal that has not been blocked by the matrix. Since this useless part of the burst will reach the next node it can cause false optical signal detections and therefore additional information such as jam sequence might need to be added.

In our study we assume that the feed-back buffer emulates $N$ output feed-forward buffers, each one operating with 8 optical channels, where $N$ is equal to the number of output ports. The number of delay lines is between 1 and 4 depending on the simulation. The provided delays are linearly increasing with a basic delay unit equal to $32 \mu \mathrm{s}$, which corresponds to the mean burst duration.

In Fig. 6 we show the results of BLP for different buffer size and number of wavelengths as a function of $T$. We see that even with one FDL used there is no significant gain in the performance when increasing $T$. It is due to the fact that the buffer itself introduces some variable preemption window and therefore no additional preemption offset in the input FDU is necessary. This also explains why, even with $T$ equal to 0 , the results of $B L P_{H P}$ are much lower than $B L P_{L P}$ and $B L P_{\text {Total }}$. Therefore the length of the input FDU and its resulting delay can be reduced. Note that the control architecture still keeps the control packets in core nodes waiting for the transmission of the bursts in order to avoid signaling complexity if a preemption occurs.

Finally, we can observe that application of FDLs decreases blocking probability of LP bursts, In particular, in the system with 32 wavelengths and only 1 FDL the $B L P_{L P}$ can be below $10^{-4}$ in a node.

\section{B. Network scenario}

In the network scenario, we consider three different topologies (see Figure 7): one regular topology consisting of a 25nodes Manhattan street network (with a nodal degree of 4), and two real topology consisting of the NSFNet topology of 15 nodes and 22 links, which represents an America backbone

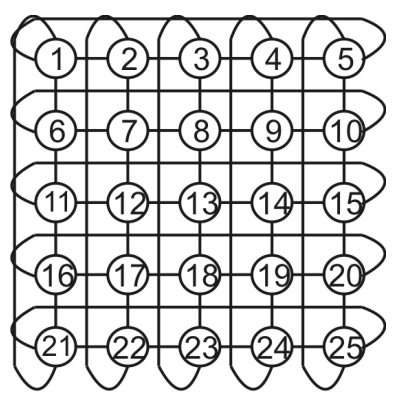

(a)

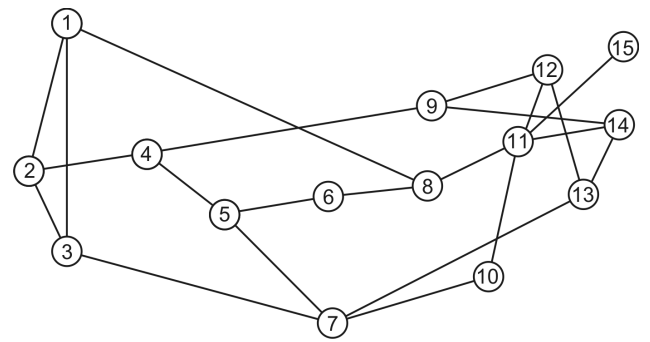

(b)

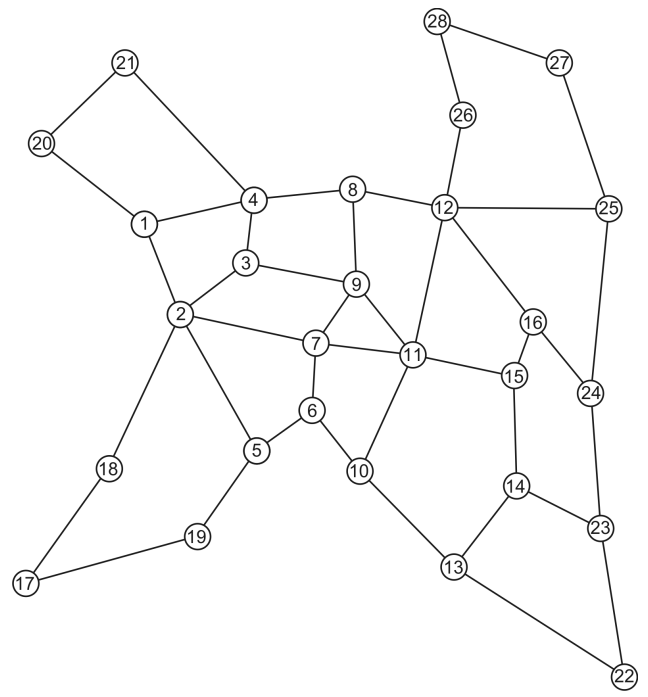

(c)

Fig. 7. a) Manhattan topology, b) NSFNet topology, c) EON topology.

network, and the EON (European Optical Network) topology with 28 nodes and 39 links.

We assume each node is both an edge and a core bufferless node capable of generating bursts destined to any other nodes. The traffic is uniformly distributed between nodes. We assume each edge node offers the same amount of traffic to the network. In this network context, the offered traffic is normalized to the transmission bitrate and expressed in Erlangs, where one Erlang corresponds to an amount of traffic that occupies an entire wavelength. For example 51.2 Erlangs mean that each edge node generates $512 \mathrm{Gbps}$, being $10 \mathrm{Gbps}$ the bitrate of each wavelength. The value of $T$ is set to $16 \mathrm{~km}$ which corresponds to 2.5 times the average burst duration.

The rest of configuration parameters is the same as in Section IV-A. 


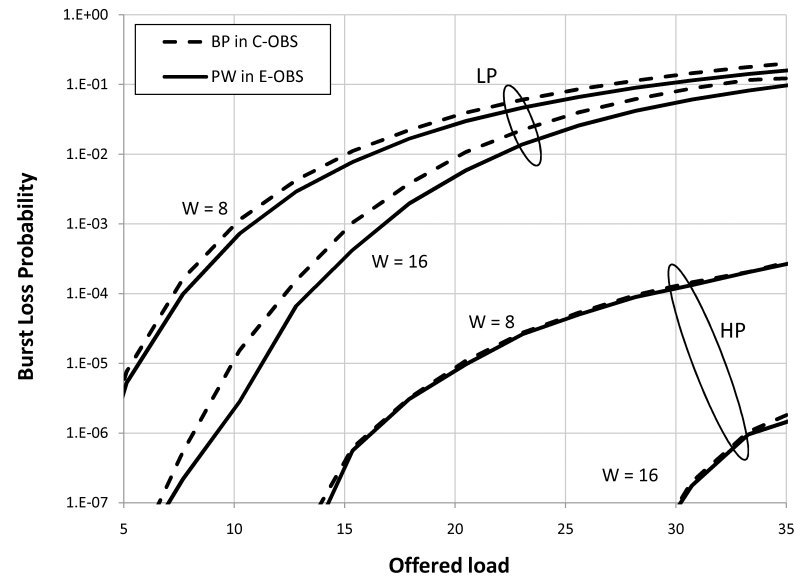

(a)

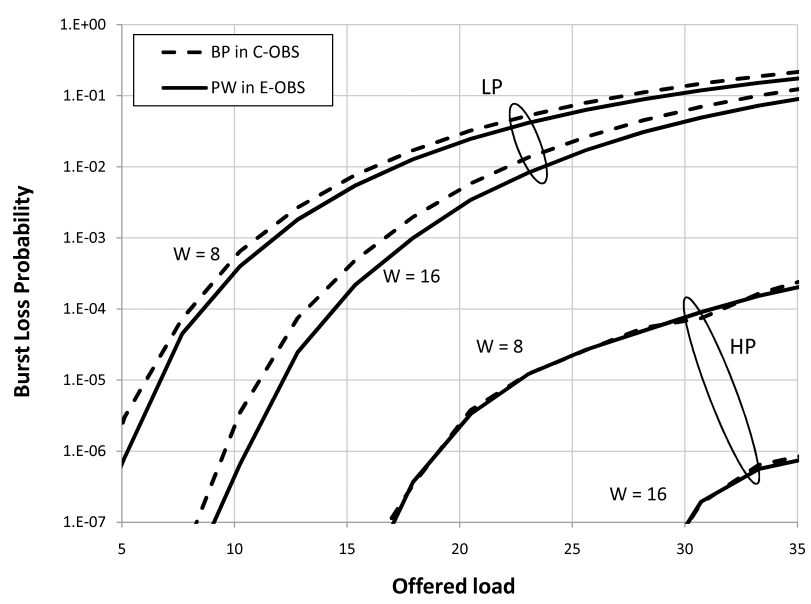

(b)

Fig. 8. Burst loss probability for LP and HP traffic comparing Burst Preemption (BP) and Preemption Window (PW) mechanisms in a) Manhattan topology, and b) NSFNet topology.

In Figure 8 and Figure 9, we compare the classical Burst Preemption (BP) applied in the C-OBS architecture and the Preemption Window $(\mathrm{PW})$ applied in the E-OBS architecture.

In Figure 8, the comparison is in terms of $B L P_{L P}$ and $B L P_{H P}$ considering Manhattan and NSFNet topologies. We select few number of wavelengths $(W=8$ and $W=16)$ in order to have significant results for HP traffic. Although the considered topologies are very different, Figure 8(a) and Figure 8(b) present similar behavior. The results show that PW presents slightly better performance for LP traffic than BP. This improvement is mainly due to the absence of phantom bursts, which, as commented in Section III, is a design feature of the PW mechanism. In terms of $B L P_{H P}$, the improvement of the PW is less evident.

In Figure 9, we show another feature of the E-OBS architecture, and, consequently, of the PW mechanism in terms of class isolation. In this figure, we focus on the fairness goodness, i.e., the variation of burst loss probabilities with respect to the residual number of hops to reach the destination for different

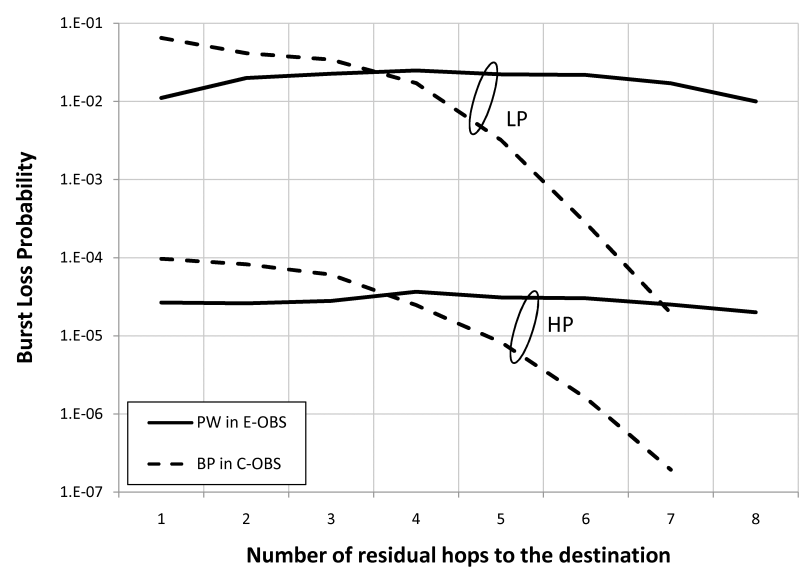

Fig. 9. Burst loss probability as a function of the number of residual hops in EON topology.

network topologies. We can see that the fairness in C-OBS is very poor. In fact, the bursts that begin their trip (i.e., with high number of residual hops, the right side of the figures) may undergo much lower losses than the bursts having just the ultimate hops to reach the destination (i.e., with few number of residual hops, the left side of the figures). On the other hand, in the E-OBS architecture each burst has the same time horizon to make the reservation of resources since the offset times, which are determined by the length of FDU, are the same. The results presented in the figure confirm this ability.

\section{CONClusion}

In this paper we studied the Preemption Window (PW) mechanism applied in the offset-time emulated OBS (E-OBS) architecture for efficient preemption-based QoS support. This E-OBS architecture applies a fiber delay unit at the input port of core nodes in order to emulate conventional offset-times. The essential part of proposed architecture is that PW allows for preemption of a low priority burst only in specific period when the burst has not reached the output link. The mechanism is also responsible for transmitting the control packet and the burst simultaneously in such a way that there is not separation between them in a link. Thanks to these rules the well-known problem of phantom burst is eliminated.

Obtained simulation results show that in a bufferless OBS node, the performance of the PW mechanism is like of the conventional burst preemption. Although the study was done for the full-burst preemption principle, the considered solution can be used with any other preemption scheme like e.g. with the burst segmentation. In the buffered node scenario, the application of FDLs decreases substantially the blocking probability of LP bursts, while, at the same time, it needs shorter fiber delay units at the input port of the core nodes.

Finally, in the network scenario, PW in E-OBS architecture surpasses the performance of the classical burst preemption applied to conventional OBS architecture. The absence of phantom bursts reduces the overall network load and thus there 
is more room for low priority traffic. Moreover, considered EOBS architecture does not experience the offsets' variations what dismisses related unfairness problem in resources reservation.

\section{APPENDIX A}

\section{THE PREEMPTION RATE IN A BUFFERLESS OBS NODE}

Let $n_{\text {preempt }}$ be the number of successful preemptions, $n_{\text {lost_HP }}^{(n p)}$ and $n_{\text {lost HP }}^{(p)}$ be the number of HP bursts lost in non-preemptive (without burst preemption) and preemptive (with full burst preemption) scenarios respectively, $n_{i n} H_{P}$ be the number of incoming HP bursts, $n_{\text {in }}$ be the total number of incoming bursts and $n_{\text {out }}$ be the total number of bursts transmitted in the output in a given period of time.

Since each preemption means the acceptance of a HP burst instead of a LP burst, $n_{\text {preempt }}$ can be also interpreted as a difference between all the HP bursts lost in the non-preemptive scenario and the HP bursts lost in the preemptive scenario:

$$
n_{\text {preempt }}=n_{\text {lost_HP }}^{(n p)}-n_{\text {lost_HP }}^{(p)}
$$

Obviously:

$$
\begin{gathered}
n_{\text {lost_HP }}^{(n p)}=n_{\text {in_HP }} \cdot B_{H P}^{(n p)}, \\
n_{\text {lost_HP }}^{(p)}=n_{\text {in_HP }} \cdot B_{H P}^{(p)},
\end{gathered}
$$

where $B_{H P}^{(n p)}$ and $B_{H P}^{(p)}$ are the HP burst loss probabilities in the non-preemptive and the preemptive scenario.

From the previous equations we obtain:

$$
\begin{aligned}
n_{\text {preempt }} & =n_{\text {in_HP }} \cdot\left(B_{H P}^{(n p)}-B_{H P}^{(p)}\right) \\
& =\alpha_{H P} \cdot n_{i n} \cdot\left(B_{H P}^{(n p)}-B_{H P}^{(p)}\right),
\end{aligned}
$$

where $\alpha_{H P}$ is the HP class load ratio.

Than the preemption rate is equal to:

$$
R=\frac{n_{\text {preempt }}}{n_{\text {out }}}=\frac{\alpha_{H P} \cdot n_{\text {in }} \cdot\left(B_{H P}^{(n p)}-B_{H P}^{(p)}\right)}{n_{\text {in }} \cdot\left(1-B^{(p)}\right)} .
$$

Note, that the overall burst loss probability of the preemptive scenario $\left(B^{(p)}\right)$ and the HP burst loss probabilities in the nonpreemptive scenario $\left(B_{H P}^{(n p)}\right)$ are the same. Moreover, $B_{H P}^{(p)}$ depends only on the HP class load due to absolute class isolation.

Finally, assuming exponentially distributed burst arrivals and the Erlang B-loss formula:

$$
\operatorname{Erl}(A, W)=\frac{A^{W}}{W !}\left[\sum_{i=0}^{W} \frac{A^{i}}{i !}\right]^{-1},
$$

we can obtain the following estimation of the preemption rate in a node by the proper substitution:

$$
R=\frac{\alpha_{H P}\left[\operatorname{Erl}(\rho, W)-\operatorname{Erl}\left(\alpha_{H P} \rho, W\right)\right]}{1-\operatorname{Erl}(\rho, W)},
$$

where $\rho, \alpha_{H P}, W$ are respectively the overall traffic load, HP class load ratio and the number of wavelengths in a link and $\operatorname{Erl}(\cdot)$ is given by (9).

\section{ACKNOWLEDGMENT}

The work described in this paper was carried out with the support of the BONE-project ("Building the Future Optical Network in Europe"), a Network of Excellence funded by the European Commission through the 7th ICT-Framework Programme, and by the Spanish Ministry of Education and Science under the CATARO project (Ref. TEC2005-08051C03-01).

\section{REFERENCES}

[1] M. Klinkowski, D. Careglio, J. Solé-Pareta, "Offset-time emulated OBS control architecture", in Proceedings of 32th European Conference on Optical Communications (ECOC2006), Cannes, France, September 2006.

[2] M. Klinkowski, D. Careglio, D. Morat, J. Solé-Pareta, "Effective burst preemption in OBS network", in Proceedings of 2006 IEEE International Workshop on High Performance Switching and Routing (HPSR 2006), Poznan, Poland, June 2006.

[3] C. Qiao and M. Yoo, "Optical burst switching (OBS) - a new paradigm for an optical Internet", J. High Speed Networks, vol. 8, no. 1, pp. 69-84, Mar. 1999.

[4] S.J. Ben Yoo, "Optical packet and burst switching technologies for the future photonic Internet", IEEE/OSA J. Lightwave Technol., vol. 24, no. 12, pp. 4468-4492, Dec. 2006.

[5] M. Yoo, C. Qiao, S. Dixit, "Optical burst switching for service differentiation in the next-generation optical Internet", IEEE Communications Magazine, vol. 39, no. 2, pp. 98-104, Feb. 2001.

[6] M. Klinkowski, D. Careglio, S. Spadaro, J. Solé-Pareta, "Impact of Burst Length Differentiation on QoS performance in OBS networks", in Proc. ICTON 2005, Barcelona, Spain, Jul. 2005.

[7] C. Gauger, "Trends in Optical Burst Switching", in Proc. of SPIE ITCOM 2003, Orlando, FL, USA, Sep. 2003.

[8] F. Callegati, W. Cerroni, G. Muretto, C. Raffaelli, P. Zaffoni, "QoS Routing in DWDM Optical Packet Networks", in Proc. of QofIS 2004, Barcelona, Spain, September 2004.

[9] J. Aracil et al."Research in Optical Burst Switching within the ePhoton/ONe Network of Excellence", Optical Switching and Networking, vol. 4, no. 1, pp. 1-19, February 2007.

[10] Y. Xiong, M. Vanderhoute and C. Cankaya, "Control architecture in optical burst-switched WDM networks", IEEE J. Select Areas Commun., vol. 18, no. 10, pp. 1838-1851, Oct. 2000.

[11] Compact Time Delay Coil, http://www.newport.com/, 2008.

[12] Fiber Delay Coil, http://www.generalphotonics.com/DelayCoil.htm, 2008.

[13] A. Al Amin et al., "40/10 Gbps bit-rate transparent burst switching and contention resolving wavelength conversion in an optical router prototype", in Proc. ECOC 2006, Cannes, France, Oct. 2006.

[14] M. Klinkowski, D. Careglio, J. Solé-Pareta, "Comparison of conventional and offset time-emulated optical burst switching architectures", in Proc. ICTON2006, Nottingham, UK, June 2006.

[15] A. Kaheel, H. Alnuweiri, "A strict priority scheme for quality-of service provisioning in optical burst switching networks", in Proc. ISCC 2003, Antalya, Turkey, Jun. 2003.

[16] V. M. Vokkarane and J. P. Jue, "Prioritized burst segmentation and composite burst-assembly techniques for QoS support in optical burst switched networks", IEEE J. Select. Areas Commun., vol. 21, no. 7, pp. 1198-1209, Sep. 2003.

[17] M. Izal, J. Aracil, "On the influence of self-similarity on optical burst switching traffic", in Proc. of Globecom 2002, Taipei, Taiwan, pp. 23202324, Nov. 2002

[18] X. Yu, J. Li, X. Cao, Y. Chen, C. Qiao, "Traffic statistics and performance evaluation in optical burst switched networks", IEEE Journal of Lightwave Technology, vol. 22, no. 12, pp. 2722-2738, Dec. 2004.

[19] D.K. Hunter, M.C. Chia, I. Andonovic,, "Buffering in Optical Packet Switches", IEEE Journal of Lightwave Technology, vol. 16, no. 12, pp. 2081-2094, Dec. 1998 